\title{
Publisher's Note: Difference between memory and prediction in linear recurrent networks
}

[Phys. Rev. E 96, 032308 (2017)]

Sarah Marzen

Q (Received 23 October 2018; published 29 October 2018)

DOI: 10.1103/PhysRevE.98.049902

This paper was published online on 11 September 2017 with minor errors in the text and a few equations. The paper has been corrected as of 22 October 2018. The text and equations are incorrect in the printed version of the journal. 